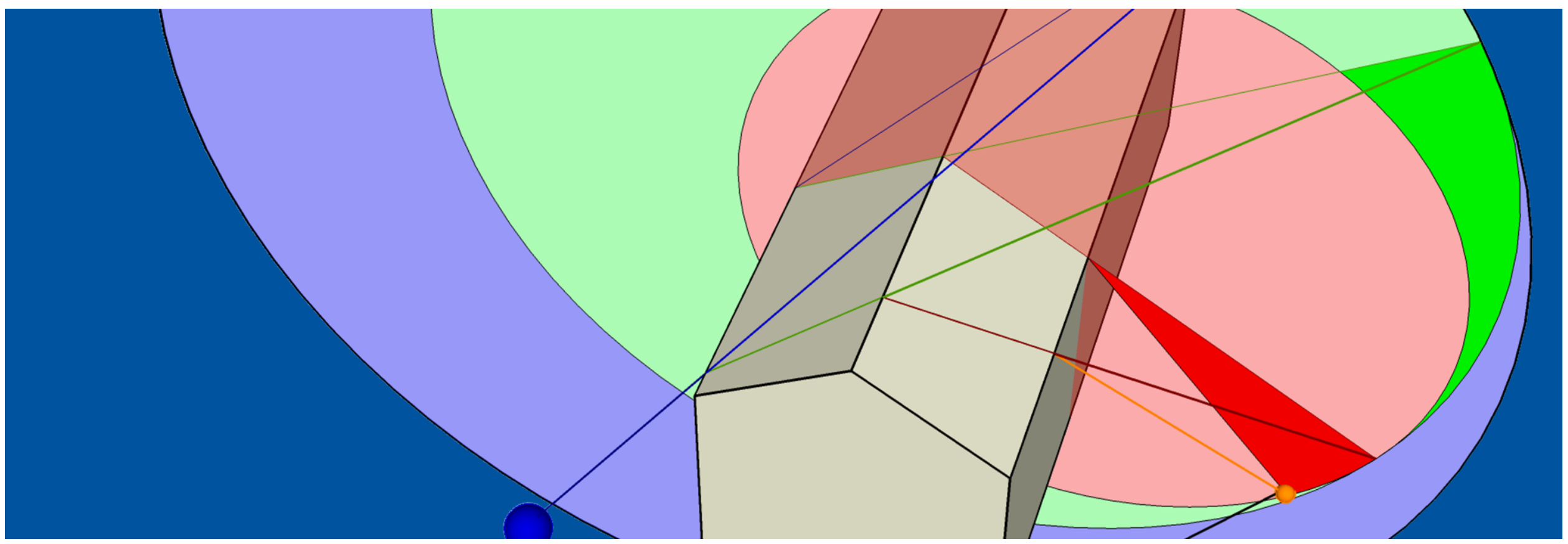

\title{
Geometry-based diffraction auralization for real- time applications in environmental noise
}

Jonas Stienen and Michael Vorländer

Institute of Technical Acoustics (ITA)

RWTH Aachen University
Presentation slides and media files are copyright protected and licensed under CC BY ND 4.0 


\section{Project overview}

\section{Auralization of Urban Environments - Real-time simulation of diffraction}

Framework for research

- Funded by DFG (German research foundation)

- 3 years duration

- Currently half-time

Objectives

- Advance auralization for urban scenarios

- Perceptual evaluation

Applications in Virtual Reality

- CAVE systems

- HMDs
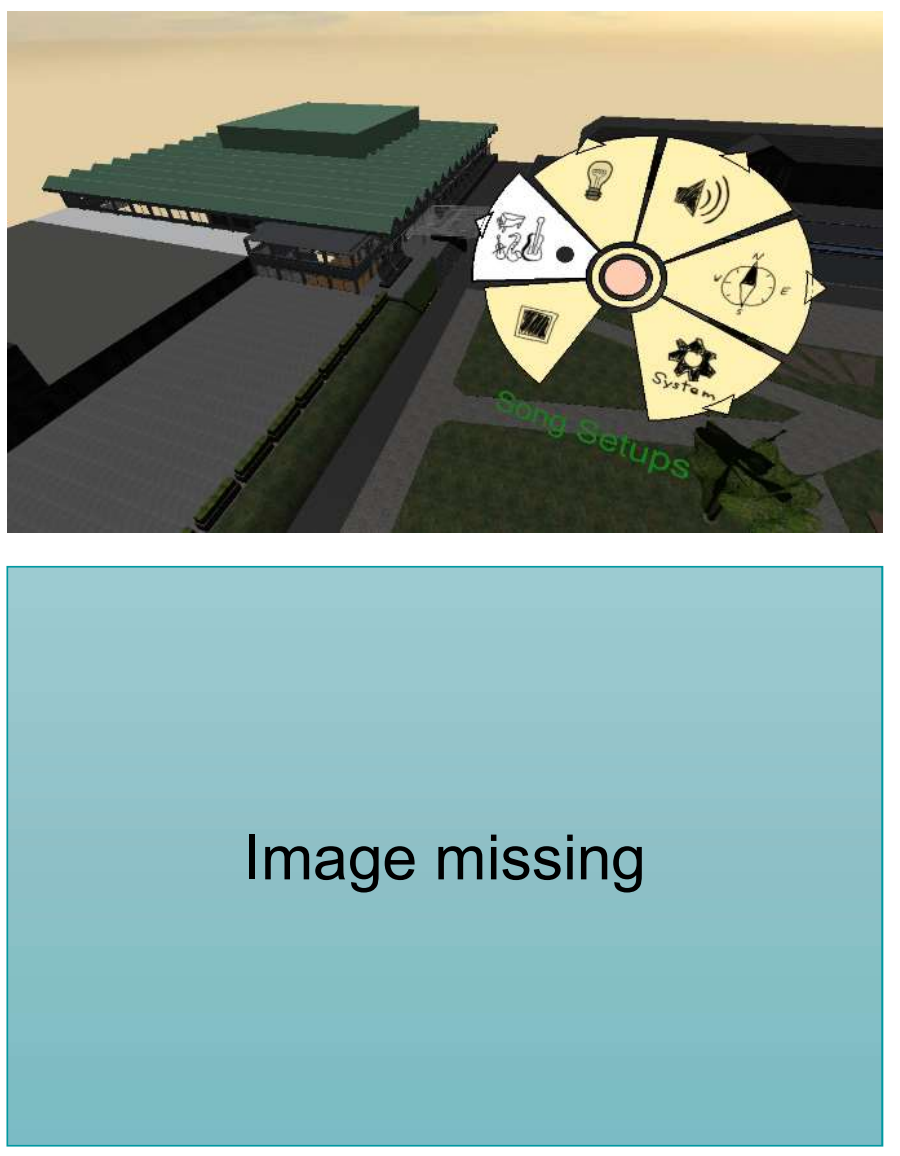


\section{From room acoustics to outdoor noise}

Room acoustics simulation and auralization

- Enclosed space

- Strong diffuse sound field component

- Plausible also without diffraction

- Edge diffraction improves accuracy

- DSP: block-base uniform or non-uniform partitioned convolution of RIR

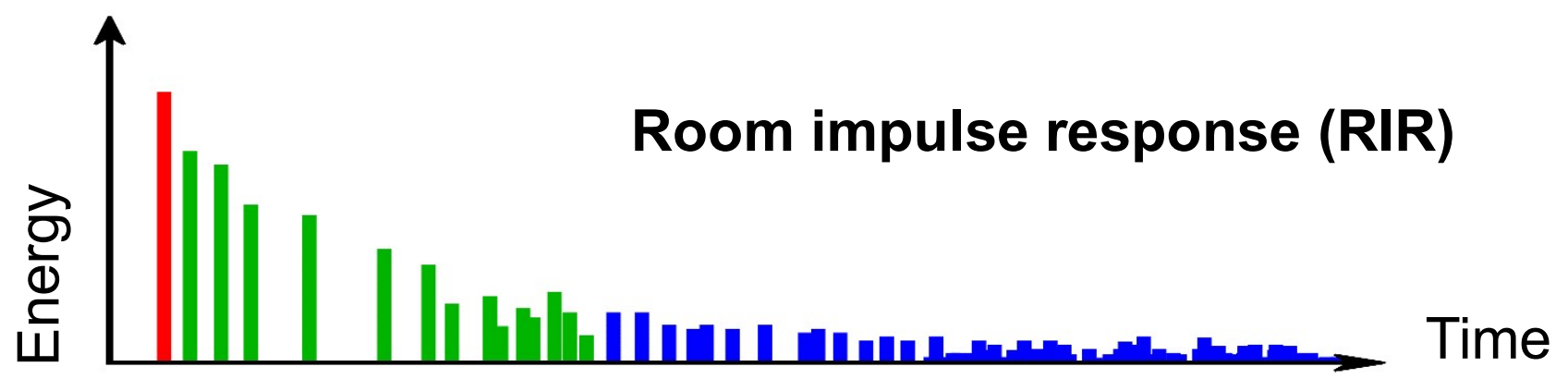

\section{early part late part}

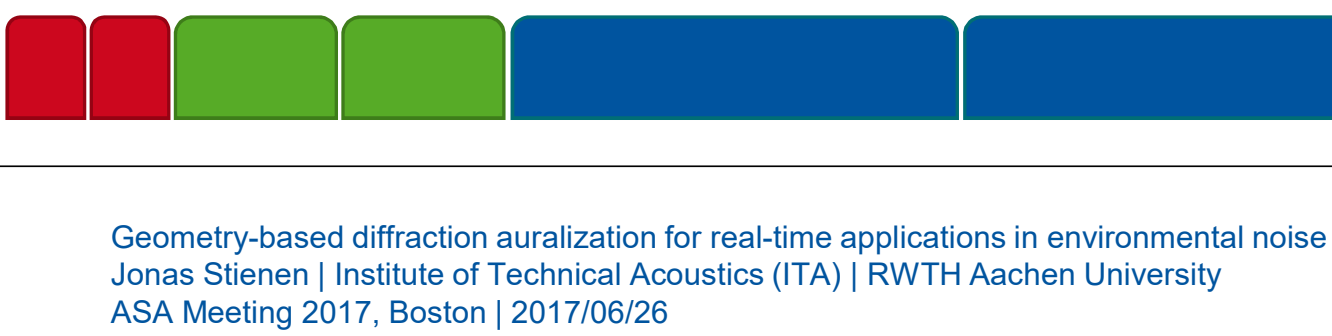

Filter segmentation 


\section{From room acoustics to outdoor noise}

Outdoor noise

- Open space

- No diffuse sound field

- Sparse wavefront arrival density

- Plausibility requires diffraction and dynamics

- Continuous sound field only with combined higher orders of reflection \& diffraction
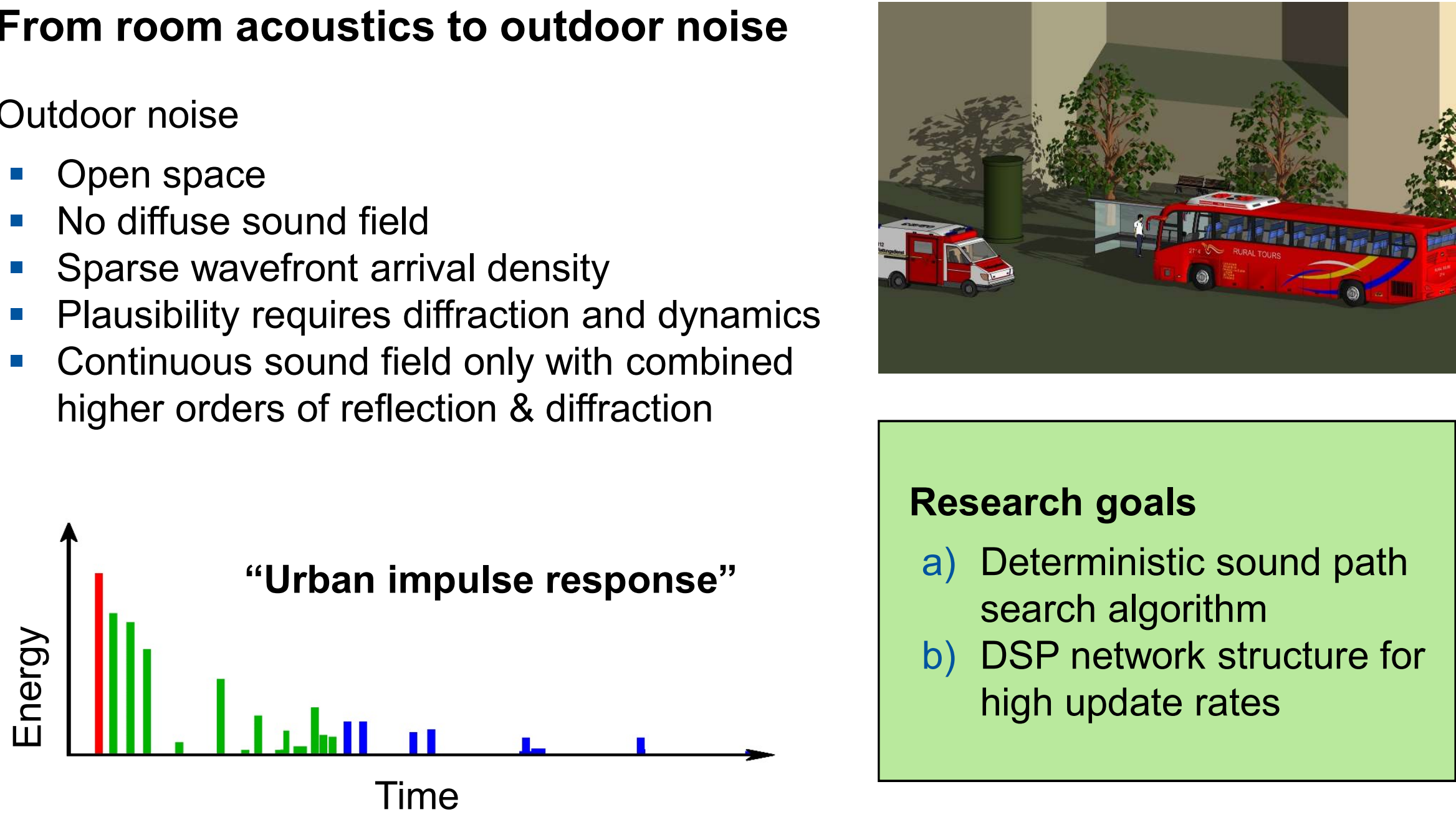


\section{Principles of Geometrical Acoustics}

Fermat's principle

- Follow wave front along shortest path

- Off flat boundaries as a mirror source

- Over edge via aperture point

Higher order simulation

- Further reflecting surfaces create a new wave front

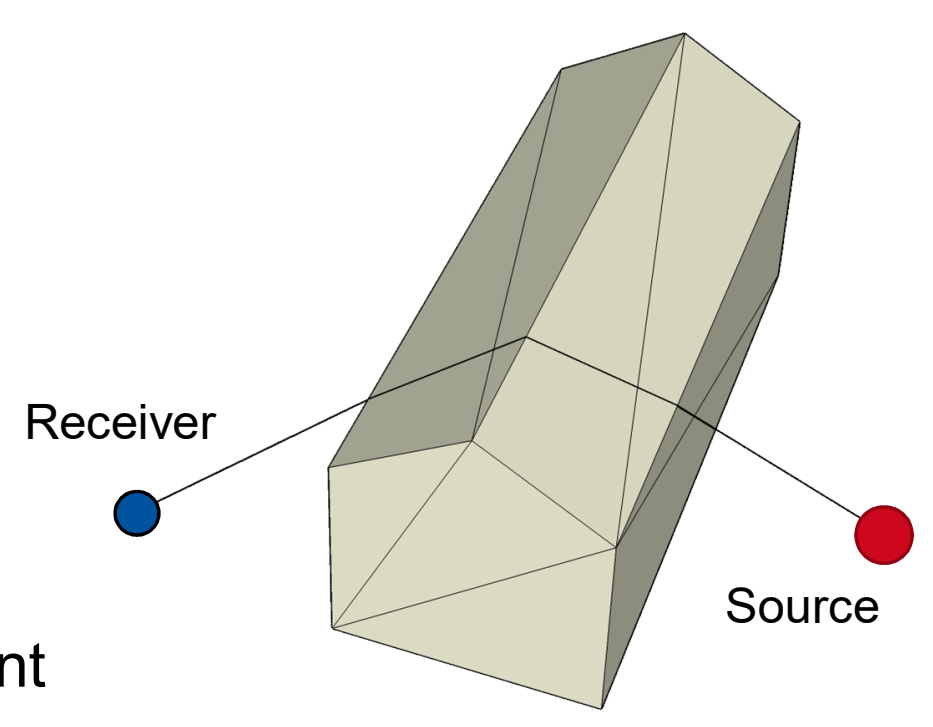

- Further diffracting edges create a new wave front

- Complexity is exponential

Algorithm

- Mirror-based image source construction [Allen \& Berkley 1979]

- Rotation-base diffraction source construction

- Modified audibility test

Allen, J. B. \& Berkley, D. A., Image method for efficiently simulating small-room acoustics, J. Acoust. Soc. Am., 1979 
Deterministic sound propagation path search algorithm

\section{Face illumination}

Gradient algorithm setup

- Preparation order $=4$

- Combination order $=4$

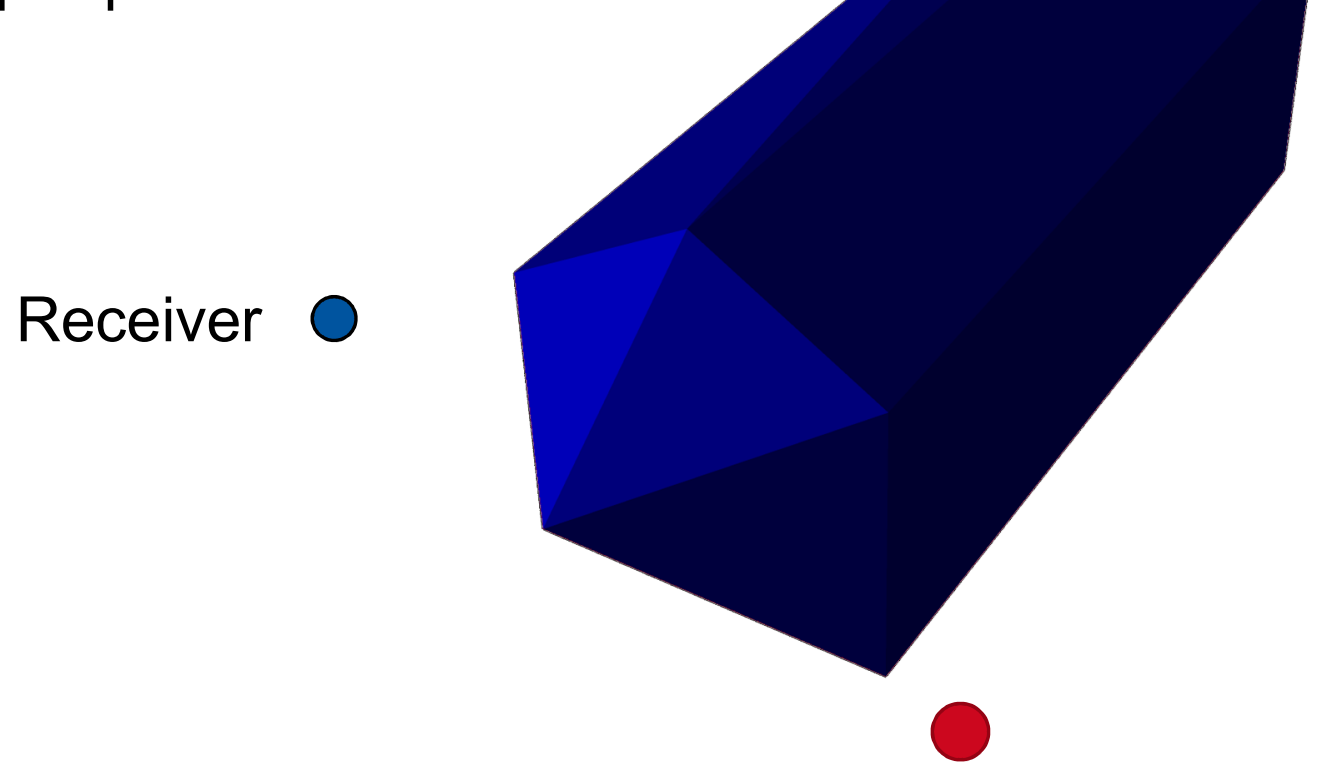

Source 


\section{Deterministic sound propagation path search algorithm}

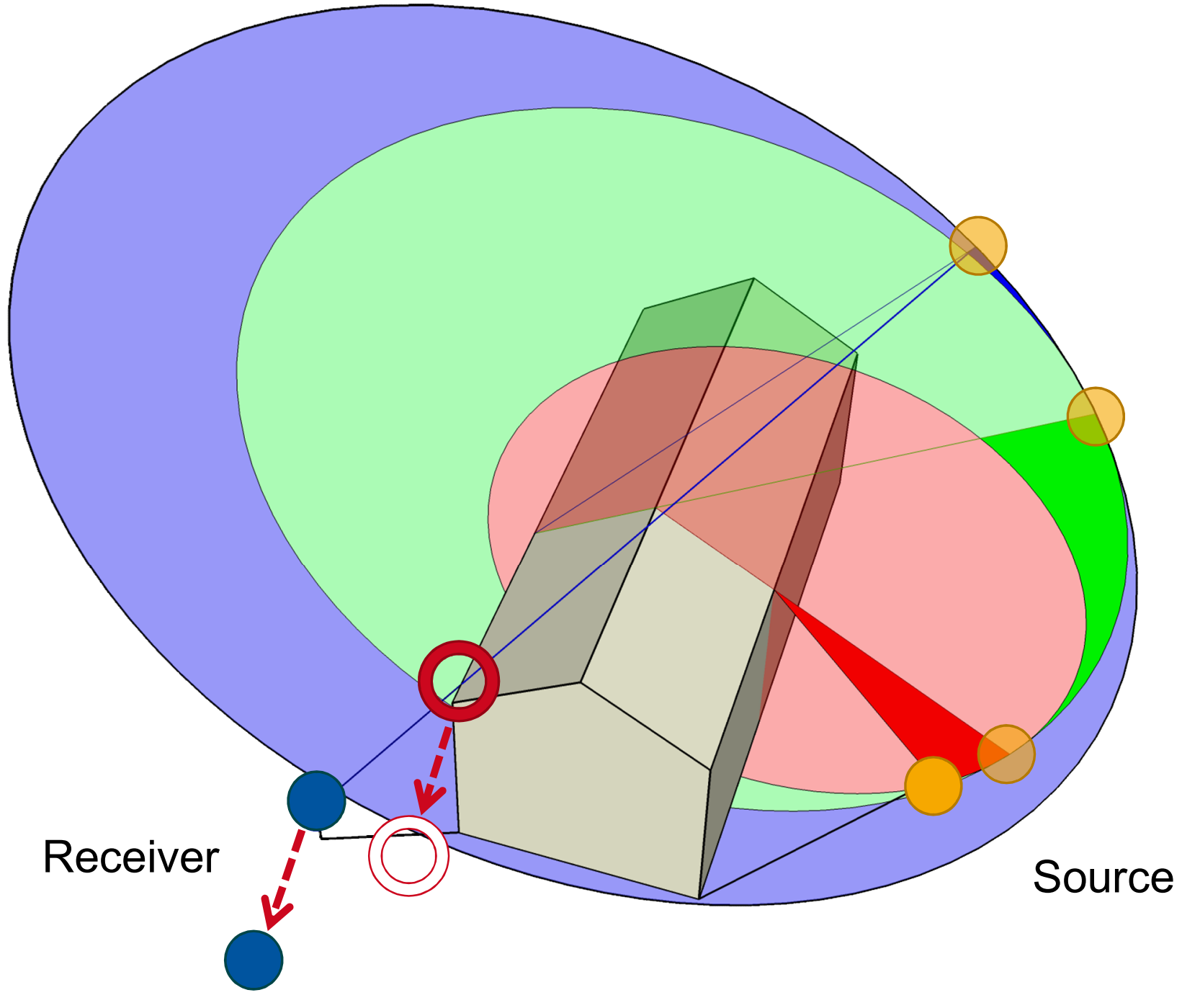




\section{Deterministic sound propagation path search algorithm}

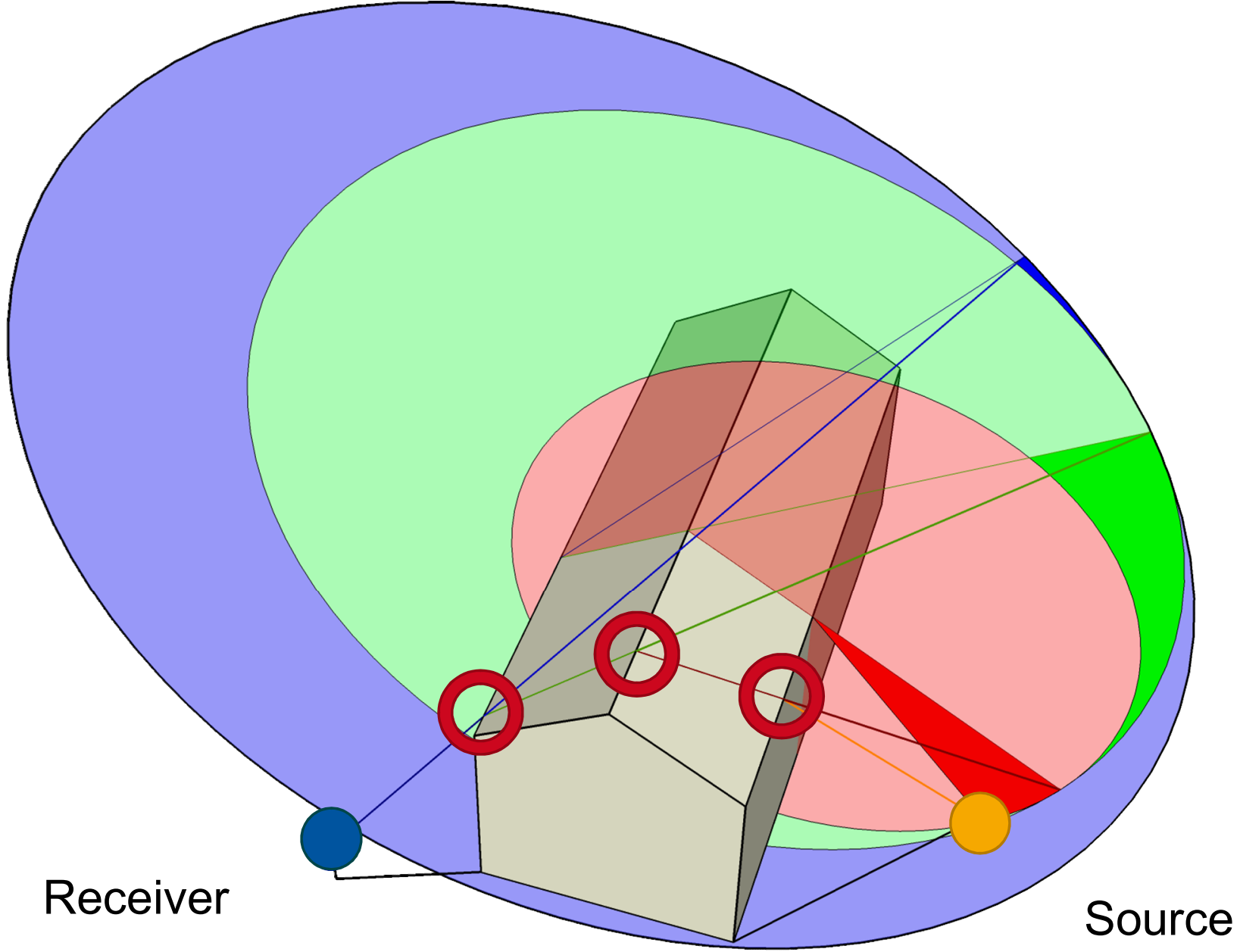


Deterministic sound propagation path search algorithm

\section{Example}

Illumination setup

- preparation order $=5$

- combination order $=5$

\section{Computation time}

- "Brute force" 5ms

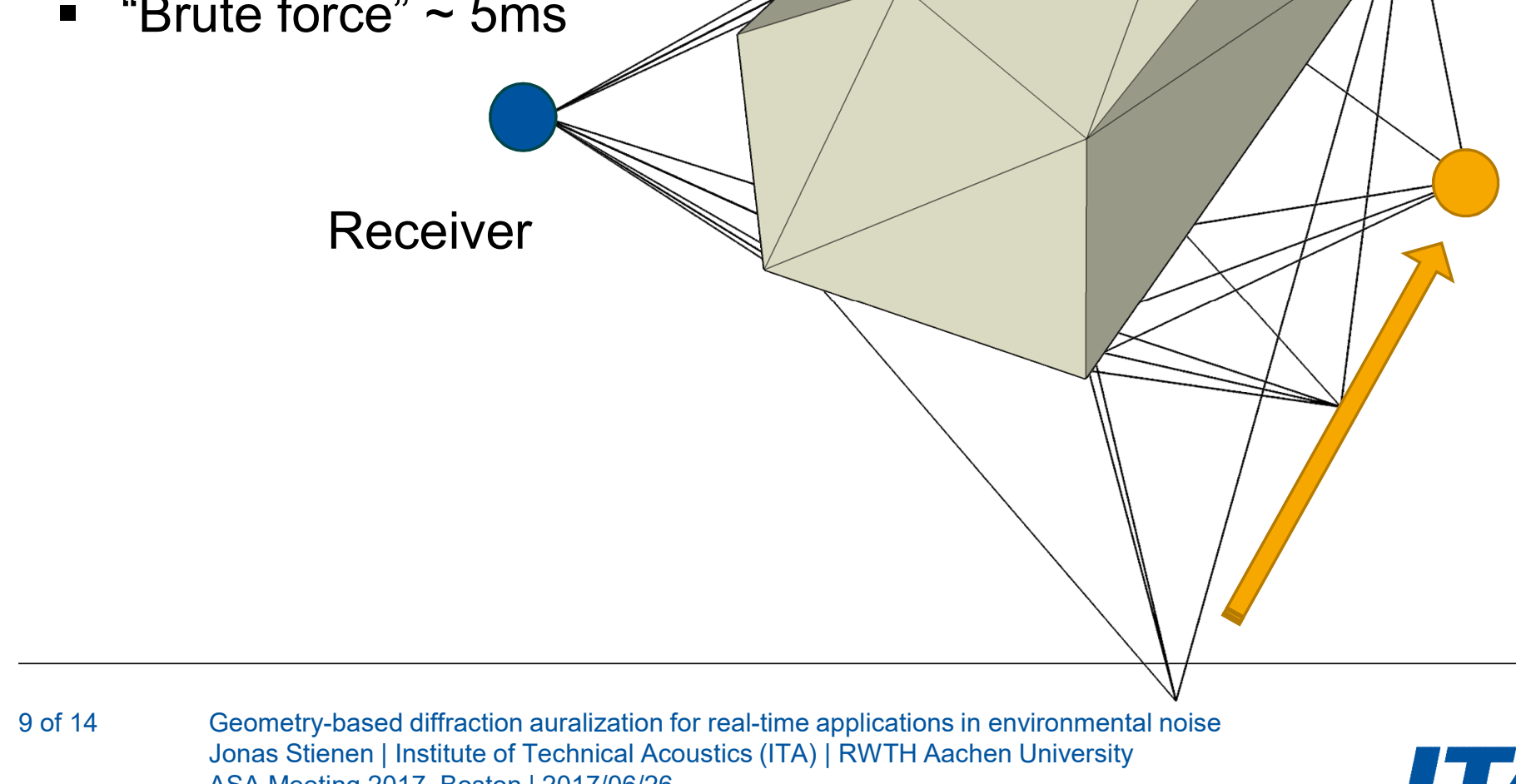


Deterministic sound propagation path search algorithm

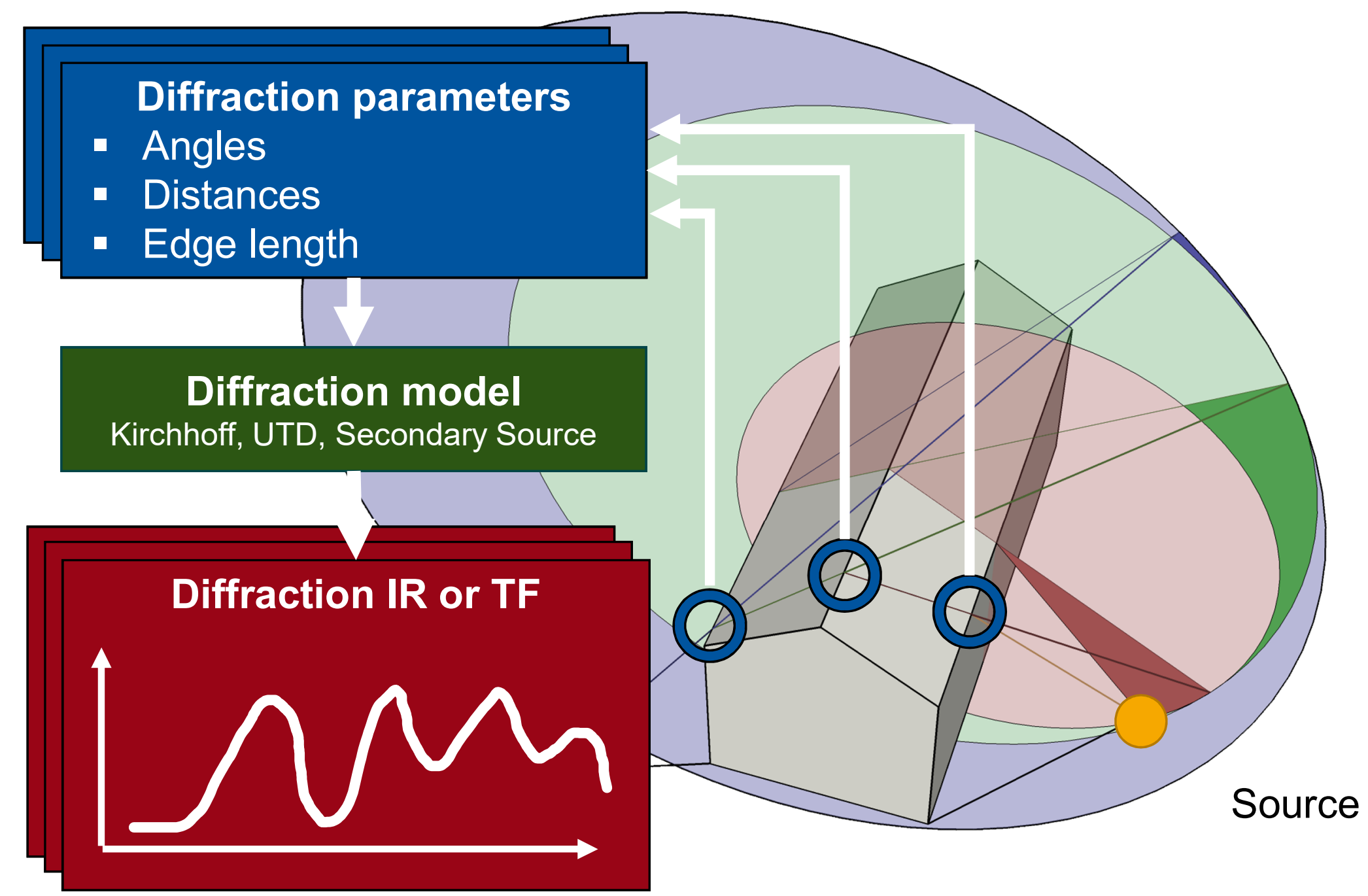


Digital signal processing network structure

Single-input multiple-output (SIMO) variable delay line

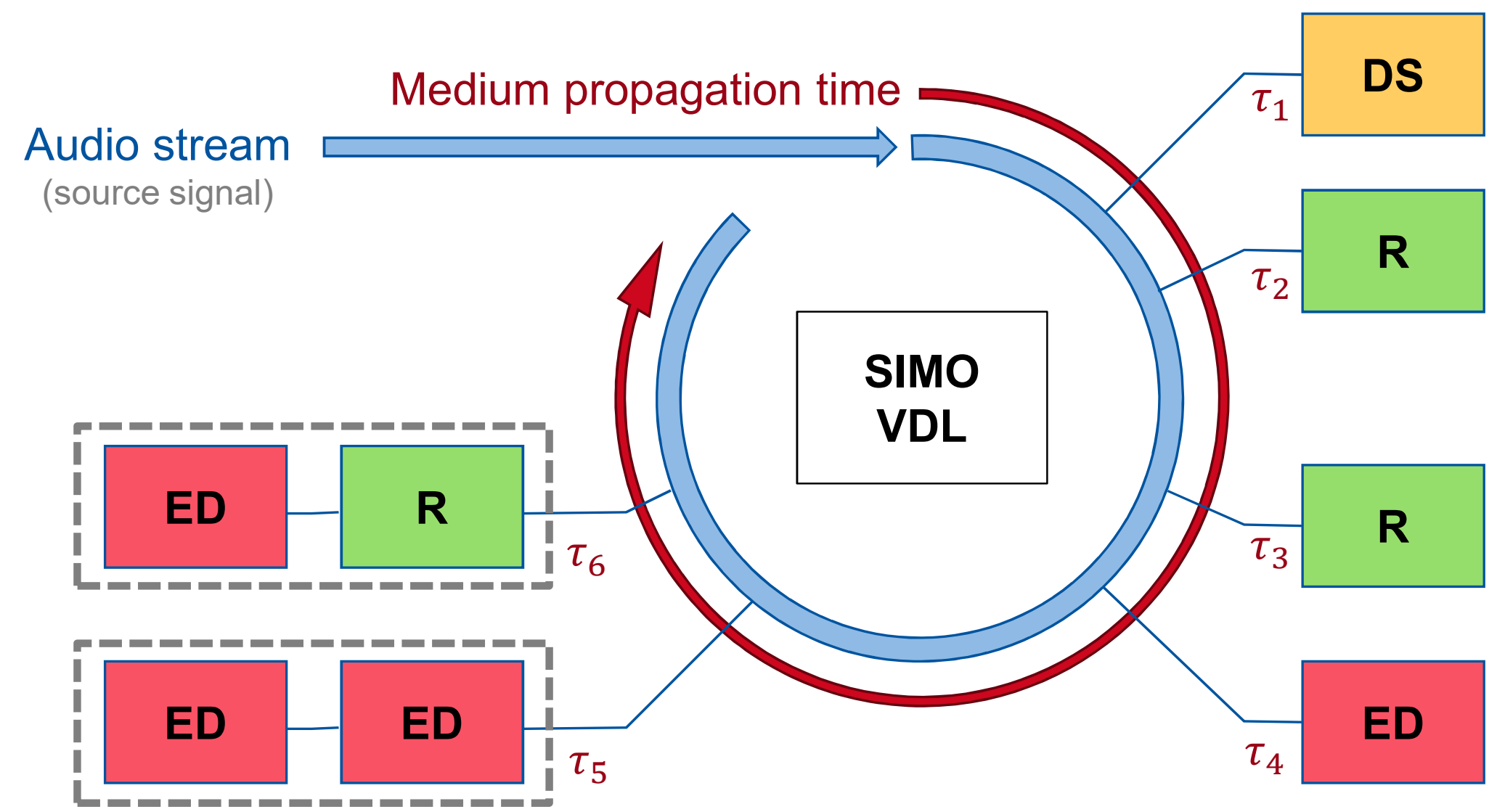




\section{Conclusion and outlook}

\section{Project: Auralization of urban environments}

Auralization of diffraction

- Diffraction important for plausibility

- Deterministic propagation path algorithm based on

- Principle: shortest path search

- Approach: rotation-based edge diffraction

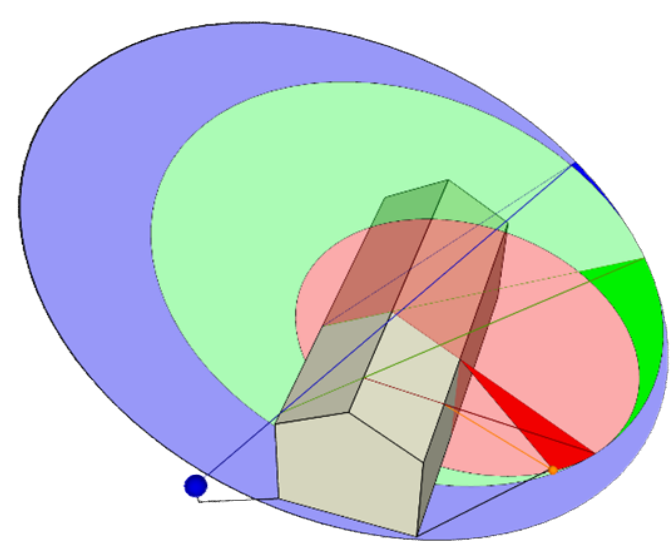

Digital signal processing

- FIR filtering not efficient

- SIMO variable delay line proposed

- Individual path rendering (enables rapid scene adaption)

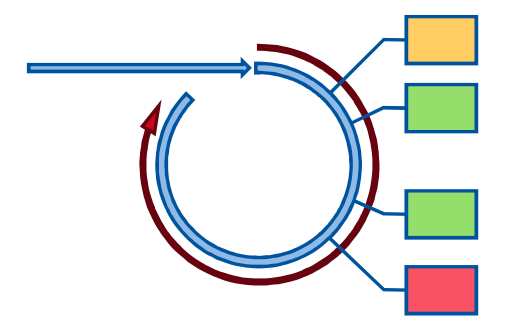

Outlook

- Finish implementation of sound diffraction models (Kirchhoff, UTD, Sec. Source)

- Integrate above for environmental noise applications with real-time auralization 


\section{Roadmap}

Public access

- Full transparency of research approach

- Accountability for research results

\section{Licensing}

- Open source (Apache License Version 2.0)

- Open access (Creative Commons)

Implementation

- C++ diffraction code (and more)

Support

- No support (except for collaboration partners)

Code repository

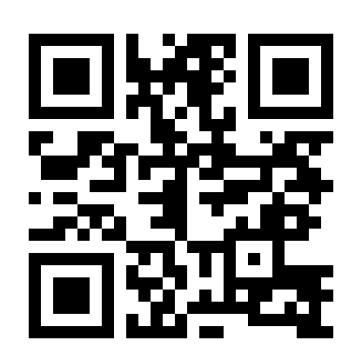

git.rwth-aachen.de/ita 


\section{Thank you for your attention}

Dipl.-Ing. Jonas Stienen

jst@akustik.rwth-aachen.de

Institute of Technical Acoustics

Virtual Acoustics Workgroup

RWTH Aachen University

web: akustik.rwth-aachen.de

repo: git.rwth-aachen.de/ita

blog: blog.rwth-aachen.de/akustik
CC BY 4.0

git.rwth-aachen.de/jst/2017_asa_boston

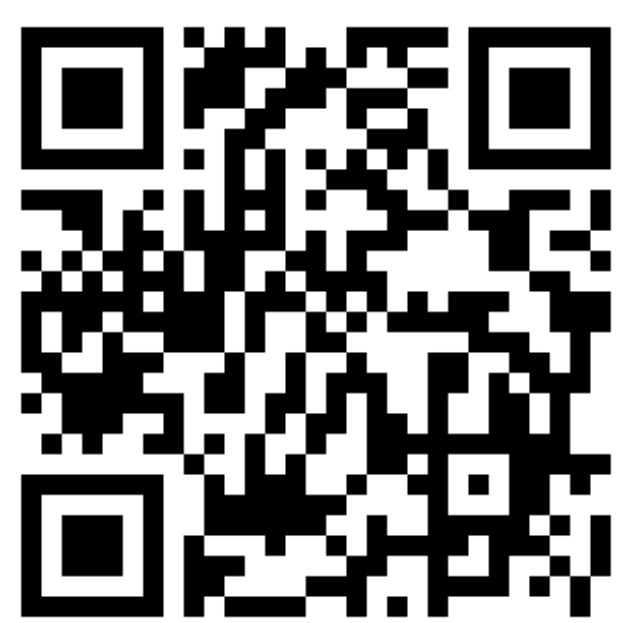




\section{Appendix: diffraction models}

\section{Kirchhoff}

- Pierce, A. D.

Diffraction of sound around corners and over wide barriers

J. Acoust. Soc. Am., 1974

\section{Uniform Thoery of Diffraction (UTD)}

- Tsingos, N.; Funkhouser, T.; Ngan, A. \& Carlbom, I.

Modeling Acoustics in Virtual Environments Using the Uniform Theory of Diffraction

Proc. of the 28th Annual Conference on Computer Graphics and Interactive Techniques, ACM, 2001

- Kouyoumjian, R. G. \& Pathak, P. H.

A uniform geometrical theory of diffraction for an edge in a perfectly conducting surface

Proc. of the IEEE, 1974

\section{Biot-Tolstoy + Medwin + Svensson (BTMS, Secondary Source)}

- Svensson, P.; Fred, R. \& Vanderkooy, J.

An analytic secondary source model of edge diffraction impulse responses

J. Acoust. Soc. Am., 1999 


\section{Appendix: rough categorization of diffraction model complexity}

\section{Benchmark results}

\begin{tabular}{l|l|l|}
\hline Model & Result length & Calculation mean (stddev) \\
\hline Kirchhoff & 33 complex frequency values & $520 \mathrm{~ns}$ (594ns) \\
\hline UTD & 33 complex frequency values & 32 us (31us) \\
\hline BTMS & 203 real values & $9.3 m s$ (848us)
\end{tabular}

